\title{
Revisión y análisis de la sífilis venérea a través de la película El expe- rimento Tuskegee / Miss Evers' boys, en el marco de la asignatura de Microbiología y Cine
}

\author{
Isabela REBELLÓN MARTíNEZ¹, Daniela SALAZAR BALCÁZAR, Claudia Rocío CASTAÑEDA RAMÍREZ², \\ Eliana OCAMPO TORO ${ }^{3}$
}

${ }^{1}$ Medicina, Pontificia Universidad Javeriana Cali, ${ }^{2}$ Departamento de Ciencias Básicas de la Salud, Pontificia Universidad Javeriana Cali, ${ }^{3}$ Hematología y Oncología Clínica, Universidad Libre Seccional Cali (Colombia).

Autor para correspondencia: Isabela Rebellón Martínez. Correo electrónico: isarebellonm@gmail.com

Recibido el 26 de enero de 2019; modificado el 11 de julio de 2019; aceptado el 15 de julio de 2019.

Cómo citar este artículo: Rebellón Martínez I, Salazar Balcázar D, Castañeda Ramírez CR, Ocampo Toro E. Revisión y análisis de la sífilis venérea a través de la película El experimento Tuskegee / Miss Evers' boys, en el marco de la asignatura de Microbiología y Cine. Rev Med Cine [Internet] 2019;15(4): 203-13.

DOI: http://dx.doi.org/10.14201/rmc2019154203213

\section{Resumen}

Alternativas innovadoras de enseñanza en el aula son fundamentales ante las limitaciones de la magistralidad. En este sentido, los desarrollos tecnológicos han permitido nuevas formas de enseñanza interactiva, en donde el Cine es un ejemplo significativo para la educación médica. A través del Cine es posible contextualizar conceptos básicos, clínicos e incluso humanistas de las enfermedades; por tanto, en este estudio se presenta una experiencia basada en cine para la enseñanza de enfermedades infecciosas y microbiología. Nosotros revisamos los aspectos históricos, epidemiológicos, microbiológicos y clínicos de la Sífilis a través de la película Miss Evers' boys, seleccionando escenas claves para cada aspecto, y comparándolas con la literatura actual. Los resultados mostraron que la película refleja elementos importantes de la evolución, diagnóstico y tratamiento de la sífilis; en donde el entendimiento por parte de los estudiantes mejora, incluso ante la ausencia de escenas sobre el desarrollo completo de la enfermedad; por consiguiente, nosotros proponemos esta estrategia como una herramienta útil y significativa para el aprendizaje en esta y otras áreas.

Palabras clave: experimento Tuskegee; sífilis; microbiología; cine.

\section{Review and analysis of venereal syphilis through the film Miss Evers' boys, within the framework of the subject of Microbiology and Cinema}

Summary

\begin{abstract}
Innovative alternatives in the classroom are essential due to the limitations of the master class. In this sense, technological advances have allowed new alternatives for interactive teaching, where Cinema is a significant example of medical education. Through Cinema it is possible to contextualize basic, clinical and even humanistic concepts of diseases; Therefore, this study presents a filmbased experience of the teaching of infectious diseases and microbiology. We review the historical, epidemiological, microbiological and clinical aspects of Syphilis through the Miss Evers' boys film, selecting key scenes for each aspect, and comparing them with current literature. The results showed that the film reflects important elements of the progress, diagnosis, and treatment of syphilis; where the understanding of the students improves, even in the absence of scenes about the complete development of the disease; therefore, we propose this strategy as a useful and meaningful tool for learning in this and other areas.
\end{abstract}

Keywords: Tuskegee experiment; syphilis; microbiology; cinema. 


\section{Planteamiento y Justificación del problema}

La educación médica en el área de la microbiología debe abarcar no sólo un enfoque básico-clínico, sino que debe permitir a los estudiantes desarrollar una actitud crítica frente al manejo de conceptos sobre enfermedades infecciosas, esto incluye tener un criterio bien fundamentado acerca de los conceptos difundidos a través de los medios de comunicación y del arte. Por lo tanto es importante que durante su formación, se promuevan estrategias pedagógicas que fomenten su capacidad de argumentación oral y escrita en temas de importancia biomédica, su capacidad de búsqueda de información científica confiable y sus habilidades argumentativas y expositivas, buscando promover un profesional de la salud, capaz de sustentar conceptos relacionados con enfermedades infecciosas, al tiempo que reconoce el papel de las diferentes manifestaciones artísticas en la difusión del conocimiento científico.

El cine como estrategia pedagógica, permite a los estudiantes comprender el mundo de los microorganismos desde diferentes perspectivas como la biológica, epidemiológica, clínica, entre otras y más allá de esto, permite descubrir diferentes aspectos históricos, sociales, artísticos, éticos, culturales y humanísticos ligados al proceso de la salud y la enfermedad en relación con las enfermedades infecciosas que pueden afectar al hombre. Esto facilita el acercamiento de los estudiantes a la microbiología entendiendo al ser humano como un sujeto integral, enlazando los conceptos teóricos con el contexto real de la sociedad en que se desenvuelven y la manera como pueden ser abordados o difundidos a través del cine.

En el contexto artístico, el cine provee un medio sensible para promover actitudes de comportamiento íntegro y ético en relación a la atención y el manejo de pacientes con enfermedades infecciosas, así como para desarrollar en los médicos en formación, actitudes de respeto y tolerancia hacia el otro.

Adicionalmente este artículo servirá de apoyo para aquellos docentes y estudiantes que quieran utilizar esta película en el proceso de enseñanza-aprendizaje del tema de sífilis.

\section{Objetivos}

Objetivo general: Revisar el tema de sífilis venérea a partir de la película Miss Evers' boys, reconociendo el cine como herramienta complementaria en el aprendizaje de la microbiología y las enfermedades infecciosas.

\section{Objetivos específicos:}

1. Identificar los aspectos históricos, epidemiológicos, microbiológicos y clínicos de sífilis venérea que ofrece la película Miss Evers' boys.

2. Ampliar a través de la revisión de la literatura científica, los aspectos históricos, epidemiológicos, microbiológicos y clínicos de sífilis venérea encontrados en la película Miss Evers' boys.

\section{Introducción}

Históricamente, el cine ha mostrado atracción por las enfermedades infecciosas, llegando a plasmar muchas de ellas desde diferentes puntos de vista. El aprendizaje microbiológico a través del cine, se ha posicionado como un recurso de mucho valor en la educación médica, convirtiéndose en un complemento a los métodos clásicos o convencionales de enseñanza ${ }^{1}$. En la actualidad son cada vez más los docentes que utilizan este recurso como parte de su forma de enseñar la Medicina, resaltando el impacto de este medio para ilustrar puntos claves referentes a la ética, los valores y las creencias, así como también facilitar al estudiante la interiorización de los conceptos básico-clínicos².

Con el surgimiento de estas películas, comenzó a plantearse la pregunta de si el cine podría ser considerado o no una mejor herramienta pedagógica que la docencia clásica. Algunos autores, como Malik S. (2013) demuestran que la simulación del aprendizaje basado en películas puede ser de gran utilidad en el entrenamiento del estudiante de Medicina y en la examinación clínica del paciente. Para esto, realizó un estudio con tres grupos, cada uno compuesto de 30 estudiantes de segundo año de Medicina. En el primer grupo (Grupo B) puso a los estudiantes media hora de una película basada en simulación de entrenamiento. El segundo grupo (grupo C), tuvo media hora de clase verbal con un entrenador. $Y$ un tercer grupo (grupo A) se usaron ambos recursos, tanto la película como también la clase verbal con el entrenador. Los resultados obtenidos se marcaron por los observadores mediante una herramienta estandarizada, y se encontró que a los grupos $B$ y A les fue significativamente mejor que a el grupo $C$. Además, el 99\% de los estudiantes reportaron que la combinación de las dos formas de enseñar era la mejor opción. Se llegó a la conclusión entonces, de que incluso media hora de una película de simulación en el entrenamiento puede mejorar el desempeño del estudiante ${ }^{3}$.

Diversos autores admiten que la educación en los estudiantes debe estar inmersa en un contexto vivo, que 
forme parte del mundo que los rodea, y es por esto que las películas son una gran herramienta para llevar gran parte de la realidad, al aula universitaria y que se construya el conocimiento 4 .

Ahora bien, reconociendo el gran valor que puede tener el cine en el aprendizaje, vale la pena recordar que generalmente las películas son realizadas con fines de entretenimiento, por tanto, las ideas contenidas en las historias cinematográficas pueden utilizarse como ejemplo o contraejemplo de la ciencia, por ello en un ambiente académico, se hace necesario revisar, analizar y comparar de manera crítica la información de las películas con la literatura científica frente al tema, para así lograr establecer un aprendizaje significativo.

\section{Concepción del diseño de estudio}

Este es un estudio de tipo cualitativo que revisa el tema de sífilis a partir de la película Miss Evers' boys, utilizando el cine como herramienta valiosa de aprendizaje en el marco de la asignatura de Microbiología y Cine, en la Pontificia Universidad Javeriana Cali.

Inicialmente se realizó una aproximación mediante la lectura, para contextualizar el papel del cine como herramienta pedagógica en la educación Médica. Luego, se hizo una revisión detallada de la película Miss Evers' boys, con el objetivo de encontrar aquellas escenas claves que sirvieran como herramienta para la comprensión de la enfermedad. Se eligieron las escenas que contuviesen cualquiera de los siguientes aspectos: historia, epidemiología, microbiología, clínica, diagnóstico y tratamiento de la enfermedad. Después, se clasificaron las escenas en cada uno de los aspectos previamente descritos, utilizando la plataforma Google Drive como mecanismo de comunicación entre los participantes. Posteriormente se realizó una revisión bibliográfica en las bases de datos Pubmed, Redalyc, WorldCat, ProQuest y Medline, con el objetivo de encontrar información que ampliara lo descrito en dichas escenas. Finalmente se describió en detalle cada una de las escenas seleccionadas y mediante reuniones presenciales del equipo de trabajo se establecieron paralelos frente a la literatura, llegando a conclusiones en relación a la validez y veracidad de dichas escenas.

\section{Elaboración del reporte de resultados}

Los datos obtenidos sobre los aspectos históricos y epidemiológicos y sobre los aspectos microbiológicos, clínicos, diagnósticos y terapéuticos se encuentran recogidos en las tablas 1 y 2 respectivamente.

Tabla 1. Aspectos históricos y epidemiológicos.

\begin{tabular}{|c|c|c|}
\hline Aspectos & Escenas & Minutos \\
\hline Históricos y Epidemiológicos & $\begin{array}{l}\text { 1. El Senado le dice a la enfermera Evers que el estudio es una especie de experimento } \\
\text { Nazi. } \\
\text { 2. El Doctor Douglas está pegando unos alfileres en el tablero de su oficina, se ve mayor } \\
\text { número de casos de sífilis en Alabama, mostrando que la enfermedad se estaba dando } \\
\text { principalmente en esa población. } \\
\text { 3. El Doctor Brodus le dice al Doctor Douglas que esa población además de ser el punto de } \\
\text { concentración de la mayoría de casos de sífilis, también lo es para pelagra, neumonía y } \\
\text { tuberculosis. } \\
\text { 4. Resistencia de los participantes para pertenecer al estudio. } \\
\text { 5. Caleb dice que no confía en el gobierno (por eso no quiere ingresar al programa de tra- } \\
\text { tamiento Tuskegee), pero la enfermera Evers le dice que, si confía en ella, y lo trata de } \\
\text { convencer para que ingrese al estudio. } \\
\text { 6. El Doctor Douglas le comenta al Doctor Brodus que el estudio debe detenerse por falta } \\
\text { de dinero. } \\
\text { 7. Los Doctores Douglas y Brodus van a solicitar ayuda económica al Servicio Público de } \\
\text { Salud de los Estados Unidos, quienes responden que lo mejor sería privar a los participan- } \\
\text { tes de un tratamiento para observar las diferencias que existían en la evolución de la } \\
\text { enfermedad en la población negra. } \\
\text { 8. El Doctor Douglas le expresa a la enfermera Evers que con el estudio él pretendía } \\
\text { demostrarles a todos que los "negros" no eran diferentes a los demás, y que le enferme- } \\
\text { dad tenía igual impacto sin importar el origen étnico. } \\
\text { 9. Willie intenta acceder a un tratamiento fuera del hospital de Tuskegee y este le es nega- } \\
\text { do por pertenecer al estudio. }\end{array}$ & $\begin{array}{l}\text { 1. } 04: 41 \\
\text { 2.09:08 } \\
\text { 3. } 11: 15 \\
\text { 4. } 13: 52,14: 47,16: 30 \\
\text { 5. } 21: 19 \\
\text { 6. } 43: 20 \\
\text { 7. } 49: 20 \\
\text { 8. } 80: 37 \\
\text { 9. } 82: 04\end{array}$ \\
\hline
\end{tabular}


Tabla 2. Aspectos microbiológicos, clínicos, diagnósticos y terapéuticos.

\begin{tabular}{|c|c|c|}
\hline Microbiológicos y Clínicos & $\begin{array}{l}\text { 1. La enfermera Evers da una explicación coloquial y general a los pacientes respecto a la } \\
\text { enfermedad; el mecanismo de acción (sexual o vertical) y los síntomas que se presentarán } \\
\text { en el futuro. } \\
\text { 2. Caleb le confiesa a la enfermera Evers que llegó a tener los síntomas descritos en el libro } \\
\text { que ella le prestó. } \\
\text { 3. Willie experimenta un dolor agudo e intenso en sus miembros inferiores mientras bai- } \\
\text { laba en la competencia. } \\
\text { 4. Hodman experimenta un episodio de psicosis acompañado de alucinaciones y delirios. } \\
\text { 5. Ben le menciona a la señorita Evers que se está quedando ciego, durante una visita que } \\
\text { ella le realiza en el hospital. } \\
\text { 6. La enfermera Evers va a visitar a Hodman a su casa y lo encuentra en un estado de psi- } \\
\text { cosis realizando una fogata, en dicha escena se pueden observar lesiones en la cara de él. } \\
\text { 7. Estando en el hospital, mientras el Doctor Douglas y Willie bailan, pueden observarse } \\
\text { lesiones en el rostro de Willie. } \\
\text { 8. Al final de la película, en una visita al cementerio, se evidencia que Willie utiliza bastón } \\
\text { para caminar. }\end{array}$ & $\begin{array}{l}\text { 1. } 26: 34 \\
\text { 2. } 33: 18 \\
\text { 3. } 73: 14 \\
\text { 4. } 86: 15 \\
\text { 5. } 90: 00 \\
\text { 6. } 92: 38 \\
\text { 7. } 100: 06 \\
\text { 8. } 115: 15\end{array}$ \\
\hline Diagnóstico & $\begin{array}{l}\text { 1. La enfermera Evers recolecta muestras de punción digital de los participantes del estu- } \\
\text { dio para realizar la prueba de Wassermann. } \\
\text { 2. El Doctor Douglas realiza punción lumbar a Willie con el objetivo de estudiar la evolu- } \\
\text { ción de la enfermedad. }\end{array}$ & $\begin{array}{l}\text { 1. } 24: 08 \\
\text { 2. } 63: 08\end{array}$ \\
\hline Tratamiento & $\begin{array}{l}\text { 1. La enfermera Evers afirma que "la mala sangre" es tratable, no un } 100 \% \text { pero hay un } \\
\text { "chance" con el mercurio. } \\
\text { 2. Los pacientes hacen un círculo entre ellos para frotarse la espalda con mercurio. La } \\
\text { enfermera Evers decía que al frotar fuerte, pasaba de ser "mala sangre a sangre caliente". } \\
\text { 3. Los representantes del gobierno de los Estados Unidos hablan con los doctores para } \\
\text { cambiar el rumbo del estudio. } \\
\text { 4. La enfermera Evers se altera con el Doctor Brodus cuando se entera que los pacientes } \\
\text { no van a recibir tratamiento, si no que van a ser solamente observados y en cambio reci- } \\
\text { birían vitaminas y aspirinas como si fuese un tratamiento. } \\
\text { 5. Los doctores y la enfermera Evers empiezan a engañar a los participantes del estudio } \\
\text { diciéndoles que van a recibir tratamiento "con inyecciones en las nalgas" pero en realidad } \\
\text { es para tomar muestras de LCR con fines investigativos. } \\
\text { 6. El Doctor Brodus le dice a la enfermera Evers que la penicilina no soluciona el daño que } \\
\text { la enfermedad ya ha causado y el Doctor Douglas comenta que este medicamento tam- } \\
\text { bién puede matarlos, porque algunos pacientes que sufren de sífilis crónica pueden des- } \\
\text { arrollar una reacción alérgica fatal llamada "Herxheimer". } \\
\text { 7. El Doctor Douglas le dice a la enfermera Evers que la penicilina es buena en los casos de } \\
\text { Sífilis primaria y secundaria y que por eso Caleb tuvo suerte de curarse. } \\
\text { 8. Visita de la enfermera Evers a la casa de Hodman para la aplicación de la Penicilina, y } \\
\text { su posterior observación clínica, tiempo en el cual Hodman en su estado de psicosis ingie- } \\
\text { re } 1 \text { litro de Aguarras. } \\
\text { 9. Escena de la autopsia de Hodman, en la que el Dr Brodus le dice a la enfermera Evers } \\
\text { que la causa de su muerte fue por la intoxicación producida tras la ingesta de } 1 \mathrm{~L} \text { de agua- } \\
\text { rras. Sin embargo, el doctor reporta el hallazgo de ciertos elementos consistentes con la } \\
\text { reacción de "Herxheimer" que no pueden ser corroborados. }\end{array}$ & $\begin{array}{l}\text { 1. } 33: 58 \\
\text { 2. } 35: 26 \\
\text { 3. } 48: 10 \\
\text { 4. } 53: 34 \\
\text { 5. } 62: 48 \\
\text { 6. } 77: 04 \\
\text { 7. } 77: 30 \\
\text { 8. } 95: 23 \\
\text { 9. } 98: 18\end{array}$ \\
\hline
\end{tabular}

\section{Discusión de los resultados}

\section{Historia y epidemiología}

Desde tiempos inmemorables, los afroamericanos han sido víctimas de abusos por parte de aquellos que proclamaban ser superiores por pertenecer a una raza más pura. Esta población ha tenido que perder, pues han sido utilizados por la esclavitud durante muchos años y cuando por fin se empezó a hablar de igualdad, derechos y oportunidades, fueron utilizados para experimentos científicos, siendo los Estados Unidos de América un ejemplo de ello5,6,7. Desde antes que se realizará el experimento Tuskegee, dicha población había sido utilizada para estudios del cuerpo humano; después de la guerra civil estadounidense o guerra de secesión, se hablaba de que existían doctores que salían en la nochese decía que pertenecían al Ku Kux Klan - a secuestrar personas "negras" en el área urbana para llevarlos a hospitales, asesinarlos y utilizarlos en experimentos científicos, y 
a pesar de no haber sido comprobado esta "historia popular", algo si fue cierto, y es que las experiencias vividas por muchos de los afroamericanos como víctimas de experimentos médicos durante la esclavitud, mantuvo esta teoría como parte de su historia, y debido a que eran considerados como una "especie humana" inferior, se sentían incapaces de rehusarse a pertenecer a cualquier experimento ${ }^{5}$.

Inmediatamente después de la guerra, en Filadelfia, un grupo de personas, empezaron a robar cadáveres de los cementerios de los "negros" para luego ser llevados a escuelas de medicina y estudiar con ellos. Aún en el siglo XX, existían hospitales -principalmente del sur de América - donde los utilizaban como conejillos de Indias. Por esta razón, muchos líderes afroamericanos comenzaron a protestar sobre los abusos cometidos durante tantos años y reclamaron por sus derechos, y fue desde entonces que se crearon hospitales especiales para la población "negra”, y únicamente los profesionales afroamericanos tenían la capacidad y las habilidades de poder convencer a los "negros" para ser tratados en dichas entidades. Este miedo social impulsó la creación en 1923- nueve años antes del experimento Tuskegee del Hospital en Tuskegee, Alabama, el cual se encargaría de atender a todo el estado, incluyendo a Macon County, donde pertenecería gran parte de la población objeto de estudio 5 .

Lo anterior, contextualiza y ayuda a entender por qué esta población se ha rehusado desde siempre a pertenecer a cualquier programa de salud5,7-10, un ejemplo claro lo vemos en la película, en los minutos 00:13:52, 00:14:47, 00:16:30, donde la Enfermera Evers intenta acercarse a la población y los invita a pertenecer al programa. Inicialmente es rechazada, y solo después de varios intentos, algunos deciden acceder, aunque con miedo y desconfianza. A pesar de ello, son engañados y utilizados finalmente para aquello que siempre temieron. $Y$ en el minuto 00:21:19 Caleb expresa a la señorita Evers que no está interesado en pertenecer al estudio porque no confía en el gobierno, siendo una clara demostración del rechazo que en ese entonces existía.

El experimento Tuskegee ha sido un ejemplo de racismo médico en Estados Unidos ${ }^{5-7}$, después del estudio, salió a la luz entre la población estadounidense, que éste había sido una idea del gobierno para exterminar a la población negra, entendiéndose como un "genocidio"7,9. Y fue a partir de este hecho, que la población afroamericana se negó rotundamente a pertenecer a cualquier servicio de salud5,7,8,10. Años después, nació la sospecha entre ellos, de que la epidemia de VIH/SIDA que parecía ser más prevalente en la población afroamericana- era de nuevo, un genocidio por parte del gobierno de los Estados Unidos. Lo anterior fundamentó la teoría de lo ocurrido a partir de 1932, en el Hospital de Tuskegee, donde se generalizó y fortaleció la creencia de que los participantes del estudio habían sido inyectados con el Treponema pallidum 5,8 , e incluso se comparó con las atrocidades ocurridas durante la primera guerra mundial, como una especie de Holocausto 6 . Esto mismo es expresado en el minuto 00:4:41 por uno de los jueces, cuando dice que el estudio pareció ser similar al experimento nazi, después de que la enfermera Evers dijera que sus pacientes habían recibido la mejor atención durante el estudio.

La población afroamericana presentaba los índices más altos para enfermedades infecciosas, como pelagra, tuberculosis, y sífilis, y no infecciosas como la malnutrición ${ }^{5,11}$. Esto mismo es manifestado por el Doctor Brodus en el minuto 00:11:15, cuando le dice al Doctor Douglas que todas estas enfermedades se daban principalmente en población "negra", al igual que en el minuto 00:9:08, cuando en un mapa de los Estados Unidos, se muestra que la mayor parte de los casos de sífilis se estaban dando en Alabama, un estado conformado en su mayoría por población afroamericana ${ }^{11}$. Esto puede explicarse por el poco acceso a los servicios de salud, cimentados en el miedo que inundaba a toda esta población al considerarse un objetivo experimental en su país, y a partir del experimento Tuskegee, los índices de otras enfermedades que surgieron, como el VIH/SIDA, empezaron a ser mayores 5,8 . Por otro lado, el hecho de que esta población fuera el foco de mayor concentración de enfermedades infecciosas, de especial importancia sífilis, hizo que el Servicio de Salud Pública se interesara en ellos de manera particular, aunque existe la creencia que, no fueron los altos índices de esta enfermedad los que llamaron la atención, sino que al ser la población "negra” el $80 \%$ de Macon, Alabama, se escogió el Hospital de Tuskegee como el centro del estudio ${ }^{6}$. Por otro lado, es importante resaltar la teoría planteada por el Doctor Lewis, autor del libro The biology of the negro en el cual argumenta que, la alta prevalencia de esta enfermedad era debida a la falta de educación y de tratamiento ${ }^{6}$.

Existieron 2 factores importantes que influenciaron el desarrollo del estudio, el primero, el de mayor impacto, consistió en la idea de que existían diferencias entre "negros y blancos" en la historia natural de la 
enfermedad que podrían significar grandes descubrimientos científicos. Esto se observó en el minuto 01:20:37 cuando el Doctor Douglas le expresa a la enfermera Evers que con el estudio él pretendía demostrarles a todos que los "negros" no eran diferentes a los demás y que la enfermedad tenía igual evolución sin importar el origen étnico. Así mismo, en el minuto 00:49:20 el Doctor Douglas y el Doctor Brodus van a solicitar ayuda económica al Servicio Público de Salud de los Estados Unidos, ellos responden que lo mejor sería privar a los participantes de un tratamiento para observar las diferencias que pudiesen existir en la población negra.

El segundo factor, fue la falta de apoyo financiero, también influenciado por la gran depresión económica de 1929 , tras la primera guerra mundial ${ }^{8}$. Esto se puede inferir en el transcurso de la película teniendo en cuenta el marco histórico y geográfico en que se desarrolla. Por otra parte, algunos personajes, como el Doctor Douglas, tienen conocimiento de las limitaciones económicas para continuar con el estudio, lo cual se puede evidenciar en el minuto 00:43:10 donde él, en conversación con el Doctor Brodus le informa que el el estudio debe suspenderse.

Por otro lado, los encargados del estudio Tuskegee decidieron no explicarles a los pacientes en qué consistía la enfermedad, y utilizaron términos coloquiales como "Mala sangre" para designar la sífilis, sabiendo que este era empleado para múltiples dolencias 6,9-11. Por ende, no solo fueron privados de un tratamiento oportuno, sino también de recibir la información adecuada y ser educados al respecto. Pero eso no fue suficiente, sino que, además para aquellos que se mudaron fuera de Alabama, o que acudieron a otras instituciones de salud, el Servicio Público de Salud utilizó una red nacional para que en ninguna institución del País les fuera permitido a los participantes del estudio recibir el tratamiento antibiótico6,8,9. Existen autores que aseguran que durante el estudio algunos recibieron tratamiento, por error $u$ otras causas externas a la sífilis, aunque la información no es muy clara al respecto6,7. Esto mismo se muestra en la película en el minuto 01:22:04 cuando Wille, animado por Caleb, intenta acceder a un tratamiento fuera del hospital de Tuskegee y éste le es negado por pertenecer al estudio, siendo un motivo más a los sumados por tantos años de abusos para desconfiar del sistema de salud de su país 5,9. La vida de los afroamericanos sigue estando permeada por la historia y de esta, el Estudio Tuskegee, la más grande y triste demostración de racismo durante 4 décadas.

\section{Microbiología y manifestaciones clínicas}

Las diferentes subespecies de T. pallidum tienen como característica común el tener como reservorio natural exclusivo al ser humano. Todas las subespecies son idénticas morfológicamente, comparten el 95\% del ADN y provocan la misma respuesta serológica.

El minuto 00:26:34 de la película, es una escena clave para entender un poco - así sea en el lenguaje sencillo de la enfermera Evers- la biología de esta enfermedad. En dicha escena, la señorita Evers les comenta a los futuros participantes del estudio que la enfermedad empieza con una lesión en los genitales, indolora, causada posiblemente por la fornicación o transmitido por los padres, y que no genera mayores complicaciones pues se "duerme, pero cuando se despierta, no se puede pensar, actuar o respirar". La evolución de sífilis ocurre así, es una enfermedad de transmisión sexual o vertical, causada por la bacteria T. pallidum subespecie pallidum, que pertenece a la espiroquetas y se considera estructuralmente Gram negativa, una vez cumple con su tiempo de incubación, aparecen los primeros signos, siendo característico la presentación de una úlcera que se conoce como chancro primario o sifilítico. En la zona de inoculación se produce una infiltración mononuclear y dilatación de los vasos que termina en una vasculitis obliterativa que ocasiona una reducción en el calibre de los vasos y por ende disminuye la irrigación sanguínea resultando en necrosis que produce una depresión central con aspecto ulcerado característico de esta lesión. A esta fase se le conoce como sífilis primaria. En esta primera fase, la lesión inicia como una pápula en los genitales y luego se convierte a úlcera, que se caracteriza por ser una lesión única, indolora, húmeda e indurada que resuelve espontáneamente entre la tercera y sexta semana de evolución ${ }^{12}$.

Es interesante ver que la película, si bien muestra de manera clara la cronología en tiempo y espacio de la evolución de la enfermedad, cuando se hace un análisis detallado se puede observar que nos contextualiza en una fase de latencia o asintomática y nos lleva a un desenlace de Sífilis terciaria. No presenta signos o escenas con sífilis primaria ni secundaria, sin embargo, a manera de recuerdo "Caleb" en la escena del minuto 00:33:18 le confía a "Eunice Evers" que, en el pasado, él presentó esos síntomas, haciendo referencia a lo descrito para Sífilis primaria.

Pese a que la película no muestra la fase de sífilis secundaria, se puede inferir que ocurre como parte del proceso de enfermedad, debido a que el sistema inmune 
no soluciona por completo el foco infeccioso, lo que permite una diseminación del microorganismo. Se presenta entre la segunda y octava semana posterior a la aparición del chancro y se caracteriza por la diseminación hematógena de treponemas. Se pueden presentar síntomas constitucionales y manifestaciones específicas de tipo mucocutáneo, linfático, gastrointestinales, neurológicas y oftalmológicas.

Entre las manifestaciones mucocutáneas se encuentran rash o erupciones maculopapulares, inicialmente bilaterales y simétricas, entre 5 a $10 \mathrm{~mm}$ de diámetro, de color rosa en pacientes de piel clara, o pardo, en pacientes de piel oscura ${ }^{13}$. Un $10 \%$ de los pacientes pueden presentar erupciones superficiales secas y grisáceas en mucosas de paladar, lengua, faringe, laringe, y glande ${ }^{14}$. A nivel de mucosa genital, se observan verrugas lisas denominadas condilomas planos ${ }^{15}$.

En el sistema linfático se presentan linfadenopatías, en su mayoría cervicales y en menor frecuencia axilares e inguinales, y en el tracto gastrointestinal, ulceraciones en colon y recto ${ }^{15}$.

En el sistema neurológico, 1-2\% de los pacientes en esta fase, presentan meningismo, cefaleas y cambios en el estado de conciencia, y dentro de las alteraciones oftalmológicas, 5-10\% desarrollan iritis, uveítis anterior, posterior o panuveítis ${ }^{15}$.

En el minuto 01:03:08, Willie es el primero a quien se le realiza una punción lumbar, bajo la idea de que hacía parte del tratamiento, cuando en realidad el objetivo era estudiar la evolución natural de la enfermedad en pacientes afroamericanos. Este procedimiento no fue más que un método doloroso y peligroso por los efectos adversos que trae. En este punto de la evolución, Willie estaría dentro de la fase de latencia o asintomática característica de la enfermedad.

Al acercarse al final de la película, nos situamos en la cuarta y última década del experimento Tuskegee, donde se refleja la cronicidad de la enfermedad, observándose signos y síntomas de sífilis terciaria a través de sus personajes principales. Uno de los personajes es Willie, a través del cual se ejemplifica al paciente con neurosífilis. En el minuto 01:13:14, se observa al personaje quien, mientras bailaba muy bien, experimenta un dolor agudo e intenso en sus miembros inferiores que le hacen detener su baile por unos cuantos segundos. En esta escena, Willie representa el comienzo de la neurosífilis tabética; una enfermedad progresiva de la médula espinal que afecta entre un $2-10 \%$ de pacientes con sífilis terciaria, la cual inicia gradualmente, generalmente como un dolor agudo, intenso y punzante en miembros inferiores haciendo que la persona pierda estabilidad y deba con el tiempo caminar con los pies separados (como bien lo evidencia Willie en una de las últimas escenas, específicamente en el minuto 01:55:15 en la que debe acudir a un bastón para caminar). En términos generales, un paciente con tabes dorsalis se presentará con ataxia y pérdida de la propiocepción ${ }^{16}$.

Otro de los personajes; Bryan Hodmann, quien en el minuto 01:26:15 experimenta un episodio de psicosis acompañado de ideas delirantes que lo llevan a realizar un ritual con supuestos fines curativos. Esta escena propone al público un ejemplo del compromiso neurológico en pacientes con sífilis terciaria.

El microorganismo se localiza principalmente en los lóbulos frontales y en menor medida en los temporoparietales. Inicia como una fase preparalitica en la cual el paciente puede presetar irritabilidad, insomnio, falta de atención, confusión, alteraciones cognoscitivas, y problemas en el cálculo, habla o escritura. Por otro lado "puede aparecer delirium, crisis convulsivas, accidente cerebrovascular, afección de pares craneanos"17. La siguiente fase se caracteriza por "alteraciones del juicio crítico, ideas delirantes megalomanîacas, expansivas, euforia"17. En ocasiones el estado de ánimo puede variar hasta llevar al paciente a cuadros depresivos severos.

En el minuto 01:30:00, el personaje Ben, se encuentra hospitalizado y recibe la visita de Eunice. Durante la conversación él le manifiesta que se está quedando ciego. La ceguera es otra de las manifestaciones en sífilis terciaria, generalmente inicia afectando un solo ojo, pero prontamente afecta a ambos ${ }^{18}$. Sin embargo, es importante tener en cuenta que las afecciones oculares pueden presentarse también sífilis secundaria ${ }^{15}$.

Por otro lado, son varios los momentos finalizando la película en las que aparecen los personajes con lesiones en el rostro, como lo demuestran los minutos 01:32:38 y 01:40:06 que muestran dichas lesiones en la cara de Hodman y Willie respectivamente. Esta es otra de las características de esta fase; las lesiones granulomatosas en piel conocidas como gomas, que pueden ser múltiples o difusas, pero generalmente se presentan solitarias, varían de tamaño, oscilan desde lo imperceptible al ojo humano hasta medir $10 \mathrm{~cm}$ de diámetro y suelen localizarse en cara, mucosas y tronco. Son lesiones induradas e indoloras que en ocasiones logran ulcerarse e incluso generar perforaciones óseas ${ }^{19}$. 


\section{Diagnóstico}

En el minuto 00:24:08, la enfermera Evers colecta muestras de punción digital de los participantes del estudio para realizar una prueba diagnóstica de sífilis, para esa época el estudio era el "test de Wassermann". Esta prueba se desarrolló en 1906 por el Doctor Wasserman, basada en la extracción de tejido hepático de fetos sifilíticos ${ }^{20}$. Lo que se buscaba con esta prueba, era encontrar la presencia de anticuerpos séricos mediante una técnica de fijación de complemento ${ }^{19}$. Sin embargo, ésta generaba reacciones inespecíficas que se demostraron posteriormente en 1910, cuando el Doctor Freundenberg expresó cierta preocupación respecto a esta técnica después de haber enviado una muestra a 2 hospitales, los cuales informaron resultados diferentes entre ellos, de manera que 6 días después decide enviar la muestra a 3 hospitales más, los cuales también informaron resultados discrepantes 21 .

El mismo Wassermann participó en la discusión y protestó en contra de su prueba, contribuyendo a mejorar así la técnica que actualmente se utiliza desde 1946; la prueba VDRL, que sus siglas representan el nombre del laboratorio donde se desarrolló "Venereal disease research laboratory". Esta técnica se realiza a partir de la purificación de dos sustancias reactivas, cardiolipina y lectina, extraídas de corazón de vacunos, las cuales son utilizadas como antígenos en la realización de la prueba.

Las pruebas de diagnóstico y seguimiento se han clasificado en no treponémicas y treponémicas. Dentro de la primera clasificación se encuentran: VDRL y la prueba rápida de reagina plasmática (RPR), por ende, no son específicas y pueden generar falsos positivos. En la segunda clasificación se encuentran la prueba de absorción de anticuerpos treponémicos fluorescentes (FTAABS) y la microhemaglutinación para Treponema pallidum (MHA-TP), que son consideradas pruebas que complementan, más no confirman el diagnóstico.

Desde la época en que se describieron las prueba tanto VDRL como FTA-ABS, han sido validadas para ser aplicadas en muestras de suero y líquido cefalorraquídeo (LCR). T. pallidum invade de forma temprana el sistema nervioso central en presencia o ausencia de síntomas que sugieran neurosífilis, sin embargo, el aislamiento de este en el LCR tiene una baja sensibilidad, entre el 15 $40 \%$ y por este motivo no se utiliza. En la práctica clínica, el diagnóstico de neurosífilis se realiza con pruebas treponémicas y no treponémicas positivas en suero, asociado a síntomas neurológicos u oftalmológicos y hallazgos positivos en LCR como pleocitosis (>20/ml), elevación de proteínas $(>0.5 \mathrm{~g} / \mathrm{L})$, o pruebas positivas ya sea VDRL o FTA-Abs ${ }^{22}$.

Por otro lado, a pesar de los pocos estudios que se han realizado aplicando pruebas moleculares como la Reacción en Cadena de la Polimerasa en Tiempo Real (RT-PCR) en LCR para T. pallidum y que estos han mostrado resultados contradictorios, en el 2016 la Infectious Disease Society of America (IDSA), en conjunto con la HIV Medicine Association y la Universidad de Oxford, realizaron un estudio con el objetivo de mostrar la utilidad de la prueba RT-PCR en LCR para el diagnóstico de neurosífilis; de este pudieron concluir que a pesar de su baja sensibilidad, cercana al $45 \%$, esta prueba tenía una especificidad mayor al 90\%, sin embargo, la sensibilidad sigue siendo demasiado baja para ser utilizada en la práctica como método diagnóstico. Esta baja sensibilidad puede deberse a varios factores, uno de ellos, se debe a la inestabilidad del treponema ante la manipulación y almacenamiento de las muestras de LCR. Por otro lado, en caso de neurosífilis el LCR no es el único objetivo del treponema, si no que existen diferentes expresiones de esta, pudiendo estar en las meninges, en el globo ocular, en los vasos sanguíneos cerebrales, y el cerebro propiamente. Otro factor consiste en que, se necesitan pocas espiroquetas en el sistema nervioso central para producir neurosífilis y puede necesitar más cantidad de ésta para producir una prueba positiva. Y por último, las espiroquetas están presentes en el LCR de forma transitoria, por lo cual pueden o no coincidir como hallazgo en la muestra de punción lumbar22.

\section{Tratamiento}

Desde el siglo XIV d.C y hasta mediados del siglo $X V$ d.C se conocen datos de la existencia de la enfermedad, así como diversas formas de tratamiento que se creían era la manera de eliminarla. En aquella época se utilizaban plantas y metales, entre esos la planta del guayaco ${ }^{23-25}$ y los ungüentos o inhalaciones en "tinas de sudor" con mercurio 23,24 , este último había sido utilizado en enfermedades como la lepra, sin embargo, en cualquiera de sus presentaciones, traía consigo efectos adversos descritos como estomatitis, diarrea, sialorrea y caída de dientes ${ }^{23-24}$.

Posteriormente empezaron a utilizarse otras técnicas como los calmantes astringentes, purgantes mercuriales, inyecciones de acetato de plomo con emolientes de aceite o con mercurio para el tratamiento del chancro, técnicas quirúrgicas como la cauterización y la escisión del chancro tanto en hombres como mujeres $^{23,24}$. Además del mercurio, también se utilizaba el 
yodo, pero este no se incluía como tratamiento de la enfermedad, sino como "auxiliar terapéutico", con el objetivo de separar la albúmina del mercurio en los tejidos, permitiendo que la forma libre del metal alcanzara mayor concentración y así pudiera actuar más rápidamente 25 .

Después del desarrollo de la vacuna contra la viruela, a finales del siglo XVIII se intentó crear la vacuna contra la sífilis pero no se tuvo éxito ${ }^{25,26}$. Esto se relaciona con las características microbiológicas del T. pallidum subespecie pallidum, el cual es un microorganismo de difícil cultivo y cuyo único reservorio es el humano.

Finalmente, el tratamiento que parecía ser prometedor fue el mercurio, el cual se empleó desde finales del siglo XV hasta el siglo XX. Durante este tiempo hubo otros descubrimientos como el Salvarsan (Arsfenamida) realizado en 1905 por Paul Ehrlich y las sulfonamidas por Dogmak, en Alemania23-25. Fue solo hasta el año 1943 cuando se propone la penicilina como tratamiento de la enfermedad después de haberse utilizado por primera vez en un grupo de pacientes sifilíticos, quienes se curaron con una única dosis intramuscular ${ }^{23,25}$. Inicialmente la penicilina se utilizó en conjunto con terapia térmica, siendo esta última abandonada, al observar que no aportaba un beneficio mayor al que ya daba el tratamiento único con el antibiótico ${ }^{25}$.

En 1932 cuando se inició el estudio Tuskegee, la penicilina aún no se utilizaba como tratamiento de sífilis. En ese entonces el manejo consistía en ungüentos con mercurio, como lo muestra la película en el minuto 00:35:26; los participantes realizan un círculo y unos a otros se frotan en la espalda el ungüento con el objetivo de que la "mala sangre" se convirtiera en "sangre caliente". Y en el minuto 00:33:58, cuando la señorita Evers le comenta a Caleb que existen tratamientos para la "mala sangre" entre esos el guayaco y el mercurio, siendo este último la mejor opción, aunque no curaba la enfermedad.

El estudio toma un rumbo diferente, ya no se estudiarían los pacientes con el fin de manejar la enfermedad, sino con el objetivo de encontrar las diferencias o similitudes fisiopatológicas en la población negra frente a los hallazgos obtenidos en el estudio de Oslo realizado en la población blanca, explicado en el minuto 00:48:10. Por ello se decide privar a sus participantes del tratamiento hasta entonces utilizado, lo cual se refleja en el minuto 00:53:34 cuando la Enfermera Evers se entera que los participantes no seguirán recibiendo la terapia con ungüentos de mercurio y a cambio se les administra vitaminas y aspirinas, haciéndoles creer que estaban siendo tratados para la enfermedad. Por otra parte, el engaño también se refleja en el minuto 01:02:48 en el que a Willie se le practica una punción lumbar con fines investigativos, haciéndole creer que hacía parte del tratamiento.

Con el descubrimiento de la penicilina muchas enfermedades infecciosas pudieron ser curadas. En el minuto 01:13:17, el Doctor Brodus se refiere a la penicilina como "oro", pues esta había sido efectiva en enfermedades como la neumonía. Sin embargo, para el estudio no sería utilizada como tratamiento y bajo el argumento de los posibles efectos secundarios del antibiótico, prohibieron usarla en los participantes.

Como ya se mencionó anteriormente, en la primera mitad del siglo XX, el tratamiento de sífilis consistía en la aplicación de metales pesados y penicilina, pero en aquellos que se encontraran en la fase latente tardía y en la fase terciaria, el uso de penicilina podría traer ciertos peligros, como la reacción de "Jarisch-Herxheimer" que, al eliminar las espiroquetas, produciría daños cardiovasculares, fiebre y ser potencialmente mortal. Por esta razón, una vez publicado lo sucedido en el Estudio Tuskegee, no hubo muchas protestas y se abstenian de aplicarla en las fases tardías, aun conociendo el efecto benéfico de la penicilina en el curso de la enfermedad6. Esto mismo es expresado en el minuto 01:17:04 cuando el Doctor Douglas le dice a la Enfermera Evers que no es posible darle tratamiento con penicilina a los participantes porque existe una reacción alérgica muy grave llamada "Herxheimer" que podría ser fatal y además podría alterar los resultados de la observación de la historia natural de la enfermedad. En otra ocasión, en el minuto 01:17:30 el Doctor Brodus le explica a la enfermera Evers que Caleb tuvo suerte de curarse con la penicilina porque se encontraba en "fases iniciales de la enfermedad".

Finalmente, en el minuto 01:35:23 la enfermera Evers visita a Hodman para aplicarle la penicilina y se queda con él para observar su evolución, tiempo en el cual Hodman en su psicosis ingiere 1 litro de Aguarras, entrando en un estado crítico por lo cual, es trasladado por la enfermera al hospital y allí fallece. El Dr Brodus tras la realización de la autopsia apreciada en el minuto 01:38:18, confirma que la causa de muerte de Hodman fue por la ingesta de Aguarras, pero encontró ciertos elementos consistentes con la reacción Jarish-Herxheimer que no pueden ser corroborados.

La reacción Jarish-Herxheimer se describe como una reacción febril aguda, posterior a la administración 
de antibióticos en diferentes enfermedades causadas por espiroquetas. Se plantea que es secundaria a la destrucción de las espiroquetas, mediada por la liberación de lipoproteínas, citoquinas y complejos inmunes, posterior al tratamiento; similar a una tormenta de citoquinas.

La reacción se produce al comienzo del tratamiento, generalmente dentro de las primeras 24 o 48 horas después de recibir la dosis inicial. Las manifestaciones suelen ser generales como escalofrío, fiebre, malestar, vómitos, cefalea, artralgias, entre otras. La reacción local se da por exacerbación de las lesiones y su duración puede variar de horas a días, por lo general no reviste gravedad mayor, aunque se han reportado casos de muerte por complicaciones ${ }^{27}$.

Actualmente, se utiliza como tratamiento de la neurosífilis, la penicilina. En cualquier tratamiento puede ocurrir la reacción de Jarish-Herxheimer, la cual, generalmente es infrecuente y cuando se reporta se relaciona principalmente con Sífilis secundaria, donde hay mayor cantidad de treponemas. En otras etapas de Sífilis, es muy raro que se presente. En caso de que se presente tal reacción, el diagnóstico de ésta es clínico y se sugiere manejar con antiinflamatorios o corticoides, de acuerdo a su severidad.

\section{Conclusiones}

El cine es una herramienta pedagógica que permite relacionar de forma integral, los conocimientos adquiridos. Amplía la imaginación y conecta con la emoción a través de imágenes, sonidos y situaciones planteadas en torno a un tema particular. Por este motivo es importante continuar incentivando la creación de espacios para este tipo de actividades que permiten conocer más a fondo una problemática y comprender el contexto real en el que se desarrolla.

La película Miss Evers' Boys de manera general muestra que en sífilis se presentan varios estadios de enfermedad, aunque no presenta signos o escenas de sífilis primaria ni secundaria, sí puede apreciarse de manera clara en el tiempo los estadios de latencia y sífilis terciaria.

La prueba de Wassermann fue el primer método diagnóstico de sífilis, la cual está basada en la extracción de tejido hepático de fetos sifilíticos que mediante la técnica de fijación del complemento buscaba la presencia de anticuerpos séricos. Sin embargo, es una prueba que generó reacciones inespecíficas, siendo reemplazada por la prueba VDRL, la cual aún se usa en la actualidad además de otras pruebas más específicas como FTA-ABS, MHA-TP y RT-PCR en LCR.

A lo largo de los años, han existido múltiples formas de tratamiento, desde metales, plantas, baños y demás, hasta el descubrimiento de la penicilina que hasta hoy en el siglo XXI, sigue siendo el tratamiento de primera línea.
En la actualidad, la reacción de Jarish-Herxheimer en sífilis, es infrecuente y en caso de presentarse, esta principalmente asociada al tratamiento con penicilina en Sífilis secundaria donde se encuentra una gran cantidad de treponemas circulantes o en lesiones localizadas.

Si bien la finalidad de este artículo es con fines educativos respecto a las herramientas que ofrece la película para el estudio de la enfermedad de sífilis y no con fines éticos, vale la pena resaltar a manera de reflexión, la importancia de someter los estudios a un comité de ética reglamentado, que sea garante de los derechos humanos.

\section{Referencias}

1. García JE, Fresnadillo MJ, García E. El cine en la docencia de las enfermedades infecciosas y la microbiología clinica. Enferm Infecc Microbiol Clin. 2002; 20(8): 403-6.

2. Darbyshire D, Baker P. El cine en la formación médica. ¿Ha tenido acogida? Rev Med Cine. 2011; 7(1): 8-14.

3. Malik S, Zaheer R, Bilal M. Impact of movie-based simulation training, with or without conventional verbal demonstration on observed OSPE scores in medical undergraduates: a double control study. J Ayub Med Coll Abbottabad. 2013; 25(1-2):127-8

4. Cappelletti GL, Sabelli MJG, Tenutto MA. ¿Se puede enseñar mejor? Acerca de la relación entre el cine y la enseñanza. Rev Med Cine. 2007; 3(3):87-91.

5. Northington V. Under the Shadow of Tuskegee: Africans Americans and Health Care. American Journal of Public Health, 1997;87 (11): 1773-1778. PubMed PMID: 9366634; PubMed central PMCID: PMC1381160.

6. Reverby SM. More than fact and fiction, Cultural memory and the Tuskegee Syphilis Study. Hastings Cent Rep. 2001;31(5):22-8.

7. McCallun JM, Arekere DM, Green BL, Katz RV, Rivers BM. Awareness and knowledge of the US. Public health syphilis study at Tuskegee: Implications for biomedical research. J Health Care Poor Underserved. 2006; 17(4): 716-733.

8. Thomas SB, Quinn SC. The Tuskegee Syphilis Study, 1932 to 1972: Implications for HIV Education and AIDS Risk Education Programs in the Black Community. Am J of Public Health. 1991; 81(11): 1498-1505. PubMed PMID: 1951814; PubMed central PMCID: PMC1405662.

9. Fairchild AL, Bayer R. Uses and Abuses of Tuskegee. Science. 1999; 284 (5416):919-921.

10. Shafer JK, Usilton L, Gleeson GA. Untreated syphilis in the male negro, a prospective study of the effect on life expectancy. Public Health Rep. 1954; 69(7): 684-690.

11. Olansky S, Simpson L, Schuman SH. Enviromental factors in the Tuskegee study of untreated syphilis in the male negro. Public Health Rep. 1954; 69(7): 691-698. 12. Singh AE, Romanowski B. Syphilis: review with emphasis on clinical, epidemiologic, and some biologic features. Clin Microbiol Rev. 1999;12(2):187-209.

13. LaFond RE, Lukehart SA. Biological Basis for Syphilis. Clin Microbiol Rev. 2006;19(1):29-49.

14. Bravo TC. Sífilis: actualidad, diagnóstico y tratamiento. Rev Fac Med UNAM. 2003;46(6): 236-242.

15. Forero N, Peña MJ. Manifestaciones dermatológia de la Sífilis. Revisión de tema. Rev Med UIS, 2011; 24 (2): 217-29.

16. Emily LH, Lukehart SA. Syphilis: using a modern approaches to understand an old disease. J Clin Invest. 2011;121(12:4584-4592.

17. Quiroga F. Trastornos psiquiátricos comunes en enfermedades neurológicas. Asoc Col de Neurologia. Capítulo 8. 2013.

18. Munive M. Neurosífilis, caso clinico y revision bibliografica. Rev Med Costa Rica Centroamérica. 2009. Vol LXVI (597):95-103.

19. Dorado JS, Arellano ER, Rodriguez A. Treponemal infections. Syphilis. Medicine Rev. 2014;11(51): 2993-3043.

20. Ledermann W. Del Wassermann al VDRL en el antiguo Instituto Bacteriológico de Chile. Rev Chil Infectol. 2014;31(5): 619-624. 
21. The wasserman test for syphilis in practice. Cal State J Med. 1910;8(8):252-253. 22. Vanhaecke C, Grange P, Benhaddou N, Blanche P, Salom D, Parize P, et al. Clinical and Biological Characteristics of 40 Patients With Neurosyphilis and Evaluation of Treponema Pallidum Nested Polymerase Chain Reaction in Cerebrospinal Fluid Samples. Clin Infect Dis, 2016; 63(9):1180-1186.

23. Comerio, C. La historia de la Sífilis o ¿la Sífilis en la historia?. Rev Med Universitaria. 2012; 8(1):1-13.

24. Turnes AL. La Sífilis en la medicina, una aproximación a su historia. Edit Cient Sindicato Med Urugay. 2005:1-23.

25. Leitner RMC, Korte C, Edo D, Braga ME. Historia del tratamiento de la Sífilis. Rev Arg Derm. 2007;88(1);6-19.

26. Ledermann W. Del Wassermann al VDRL en el antiguo Instituto Bacteriológico de Chile. Rev Chil infectol. 2014;31(5): 619-624.

27. Voto J, Jeri R, Garcia U, Arias J. Reacción de Jarish-Herxheimer en neurosifilis. Informe de dos casos fatales durante el tratamiento con penicilina. Soc NeuroPsiqu Med Legal. Sesion del miércoles 07 de junio de 1950.
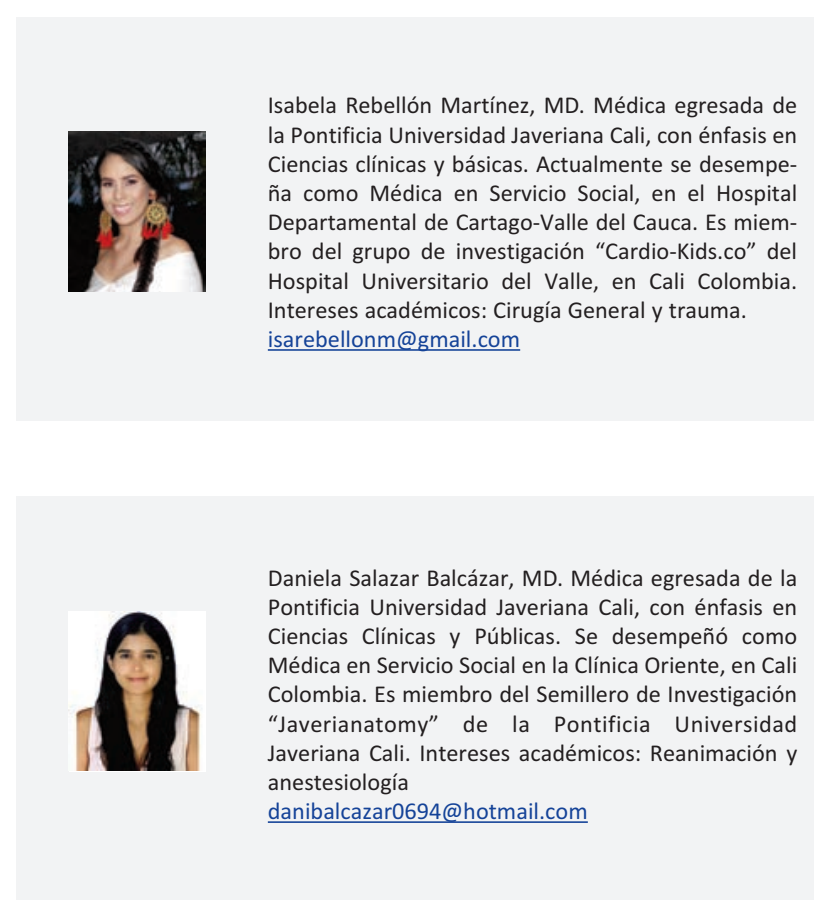

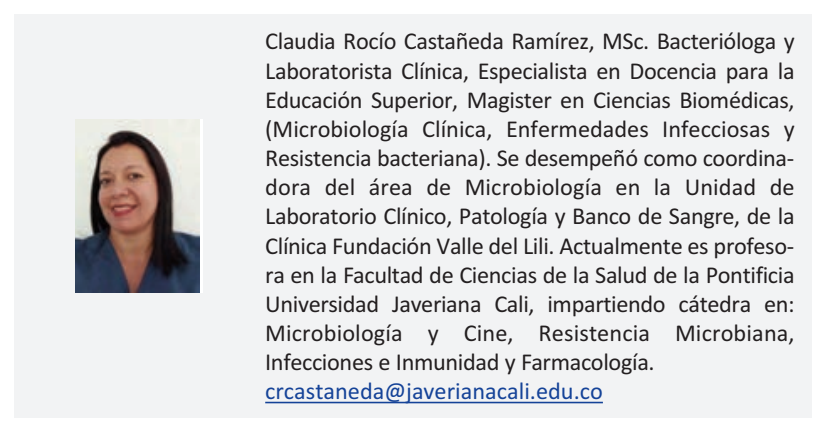

Eliana Ocampo Toro, MSc. Bacterióloga y Laboratorista Clínica, Magister en Ciencias Biomédicas, con énfasis en Inmunología y Biología Molecular. Se desempeñó como profesora en la Facultad de Ciencias de la Salud de la Pontificia Universidad Javeriana Cali, de las asignaturas: Microbiología y Cine, La Célula y Sistema Inmuno-hematopoyético. Actualmente es profesora de la Especialización de Hematología y Oncología Clínica, Universidad Libre Seccional Cali y coordinadora del área de Citometría de Flujo, Unidad de Diagnóstico Hemato-Oncológico (UDHO), Hemato-Oncólogos Cali. eliana.ocampot@unilibre.edu.co 